\title{
Magnetically regulated fragmentation of a massive, dense, and turbulent clump
}

\author{
F. Fontani ${ }^{1}$, B. Commerçon ${ }^{2}$, A. Giannetti ${ }^{3}$, M. T. Beltrán ${ }^{1}$, A. Sánchez-Monge ${ }^{4}$, L. Testi ${ }^{1,5,6}$, J. Brand $^{7}$, P. Caselli $^{8}$,

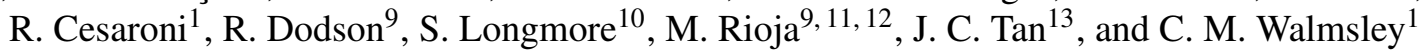

1 INAF-Osservatorio Astrofisico di Arcetri, Largo E. Fermi 5, 50125 Florence, Italy e-mail: fontani@arcetri.astro.it

2 École Normale Supérieure de Lyon, CRAL, UMR CNRS 5574, Université Lyon I, 46 allée d'Italie, 69364 Lyon Cedex 07, France

3 Max-Planck-Institut für Radioastronomie, auf dem Hügel 69, 53121 Bonn, Germany

${ }^{4}$ I. Physikalisches Institut, Universität zu Köln, Zülpicher Str. 77, 50937 Köln, Germany

5 European Southern Observatory, Karl-Schwarzschild-Str 2, 85748 Garching bei München, Germany

${ }^{6}$ Gothenburg Center for Advance Studies in Science and Technology, Chalmers University of Technology and University of Gothenburg, 41296 Gothenburg, Sweden

7 INAF-Istituto di Radioastronomia and Italian ALMA Regional Centre, via P. Gobetti 101, 40129 Bologna, Italy

8 Max-Planck-Institüt für extraterrestrische Physik, Giessenbachstrasse 1, 85748 Garching bei München, Germany

9 International Center for Radio Astronomy Research, M468, University of Western Australia, 35, Stirling Hwy, Crawley, Western Australia 6009, Australia

10 Astrophysics Research Institute, Liverpool John Moores University, Liverpool, L3 5RF, UK

11 CSIRO Astronomy and Space Science, 26 Dick Perry Avenue, Kensington WA 6151, Australia

12 Observatorio Astronomico Nacional (IGN), Alfonso XII, 3 y 5, 28014 Madrid, Spain

13 Departments of Astronomy \& Physics, University of Florida, Gainesville, FL 32611, USA

Received 31 July 2016 / Accepted 27 August 2016

\begin{abstract}
Massive stars, multiple stellar systems, and clusters are born of the gravitational collapse of massive, dense, gaseous clumps, and the way these systems form strongly depends on how the parent clump fragments into cores during collapse. Numerical simulations show that magnetic fields may be the key ingredient in regulating fragmentation. Here we present ALMA observations at $\sim 0.25^{\prime \prime}$ resolution of the thermal dust continuum emission at $\sim 278 \mathrm{GHz}$ towards a turbulent, dense, and massive clump, IRAS $16061-5048 \mathrm{c} 1$, in a very early evolutionary stage. The ALMA image shows that the clump has fragmented into many cores along a filamentary structure. We find that the number, the total mass, and the spatial distribution of the fragments are consistent with fragmentation dominated by a strong magnetic field. Our observations support the theoretical prediction that the magnetic field plays a dominant role in the fragmentation process of massive turbulent clumps.
\end{abstract}

Key words. stars: formation - submillimeter: ISM - ISM: molecules - ISM: individual objects: IRAS 16061-5048c1

\section{Introduction}

High-mass stars, multiple systems, and clusters are born from the gravitational collapse of massive, dense clumps (compact structures with $M \geq 100 M_{\odot}$, and $n\left(\mathrm{H}_{2}\right) \geq 10^{4} \mathrm{~cm}^{-3}$ ) inside large molecular clouds. Stars more massive than $8 M_{\odot}$ are expected to form either through direct accretion of material in massive cores within the clump that does not fragment further (e.g. McKee \& Tan 2003; Tan et al. 2013), or as a result of a dynamical evolution where several low-mass seeds competitively accrete matter in a highly fragmented clump (Bonnell et al. 2004). In the latter scenario, each clump forms multiple massive stars and many lower mass stars: the unlucky losers in the competitive accretion competition. There is still vigorous debate on which of these scenarios is more likely to occur, and fragmentation appears to be particularly important in this debate. Theoretical models and simulations show that the number, the mass, and the spatial distribution of the fragments depend strongly on which of the main competitors of gravity is dominant. The main physical mechanisms that oppose gravity during collapse are intrinsic turbulence, radiation feedback, and magnetic pressure (e.g. Krumholz 2006; Hennebelle et al. 2011). Feedback from nascent protostellar objects through outflows, winds, and/or expansion of ionised regions (especially from newly born massive objects) can be important in relatively evolved stages (Bate 2009), but even then seems to be of only secondary importance (Palau et al. 2013).

In a purely gravo-turbulent scenario, the collapsing clump should fragment into many cores, the number of which is comparable to the total mass divided by the Jeans mass (Dobbs et al. 2005); on the other hand, fragmentation can be suppressed by temperature enhancement that is due to the gravitational energy radiated away from the densest portion of the clump that collapses first (Krumholz 2006), or by magnetic support (Hennebelle et al. 2011). Commerçon et al. (2011) have shown that models with strong magnetic support predict fragments more massive and less numerous than those predicted by the models with weak magnetic support. The crucial parameter in their 3D simulations is $\mu=(M / \Phi) /(M / \Phi)_{\text {crit }}$, where $(M / \Phi)$ is the ratio between total mass and magnetic flux, and the critical 
value $(M / \Phi)_{\text {crit }}$, that is, the ratio at which gravity is balanced by the magnetic field (thus, for $(M / \Phi)_{\text {crit }}>1$ the magnetic field cannot prevent gravitational collapse), is given by theory (Mouschovias \& Spitzer 1976). The outcome of the simulations also depends on other initial global parameters of the clump, such as gas temperature, angular momentum, total mass, and average volume density. Once these parameters are fixed, however, the final population of cores shows a strong variation with $\mu$.

Studies of the fragmentation level in massive clumps at the earliest stages of the gravitational collapse remain limited so far. These investigations are challenging for several reasons: pristine, massive clumps are rare and typically located at distances larger than $1 \mathrm{kpc}$, hence reaching the linear resolution required for a consistent comparison with the simulations (about $1000 \mathrm{AU}$ ) requires observations with sub-arcsecond angular resolution. Furthermore, the low mass of the fragments expected in the simulations (fractions of $M_{\odot}$ ) requires extremely high sensitivities. In general, the few studies performed so far with sub-arcsecond angular resolution, or close to $\sim 1^{\prime \prime}$, reveal either low fragmentation (e.g. Palau et al. 2013; Longmore et al. 2011), or many fragments that are too massive, however, to be consistent with the gravoturbulent scenario (Bontemps et al. 2010; Zhang et al. 2015). Furthermore, comparisons with models that assume the actual physical conditions (temperature, turbulence) of the collapsing parent clump have not been published yet.

In this letter, we report on the population of fragments derived in the image of the dust thermal continuum emission at $\sim 278 \mathrm{GHz}$ obtained with the Atacama Large Millimeter Array (ALMA) towards the source IRAS 16061-5048c1, hereafter I16061c1, a massive $\left(M \sim 280 M_{\odot}\right.$, Beltrán et al. 2006; Giannetti et al. 2013) and dense (column density of $\mathrm{H}_{2}, N\left(\mathrm{H}_{2}\right)$ $1.6 \times 10^{23} \mathrm{~cm}^{-2}$ ) molecular clump located at $3.6 \mathrm{kpc}$ (Fontani et al. 2005). The clump was detected at $1.2 \mathrm{~mm}$ at low angular resolution with the Swedish-ESO Submillimeter Telescope (SEST, panel A of Fig. 1, Beltrán et al. 2006), and found to be not blended with nearby millimeter clumps, which allows a clear identification of the fragments. Its high mass and column density make it a potential site for the formation of massive stars and rich clusters, according to observational findings (Kauffmann \& Pillai 2010; Lopez-Sepulcre et al. 2010). The clump was classified as an infrared dark cloud because it was undetected in the images of the Midcourse Space Experiment (MSX) infrared satellite, although more sensitive images of the Spitzer satellite revealed infrared emission at a wavelength of $24 \mu \mathrm{m}$ (panel A in Fig. 1). Nevertheless, several observational results indicate that the possibly embedded star formation activity is at a very early stage (Sanchez-Monge et al. 2013). In particular, the depletion factor of $\mathrm{CO}$ (ratio between expected and observed abundance of $\mathrm{CO}$ ) is 12 . This provides strong evidence for the chemical youth of the clump, because the cause of depletion factors of $\mathrm{CO}$ larger than unity is the freeze-out of this molecule onto dust grains, a mechanism efficient only in cold and dense pre-stellar and young protostellar cores (Caselli et al. 1999; Emprechtinger et al. 2009; Fontani et al. 2012). The observations and data reduction procedures are presented in Sect. 2. Our results are shown in Sect. 3 and discussed in Sect. 4.

\section{Observations and data reduction}

Observations of I16061c1 with the ALMA array were performed during June, 2015. The array was in configuration C366 , with maximum baseline of $1091 \mathrm{~m}$. The phase centre was at RA (J2000): 16 $10^{\mathrm{m}} 06$ ! $^{\prime} 61$ and Dec (J2000): -50 50'29' The total integration time on source was $\sim 18 \mathrm{~min}$. The

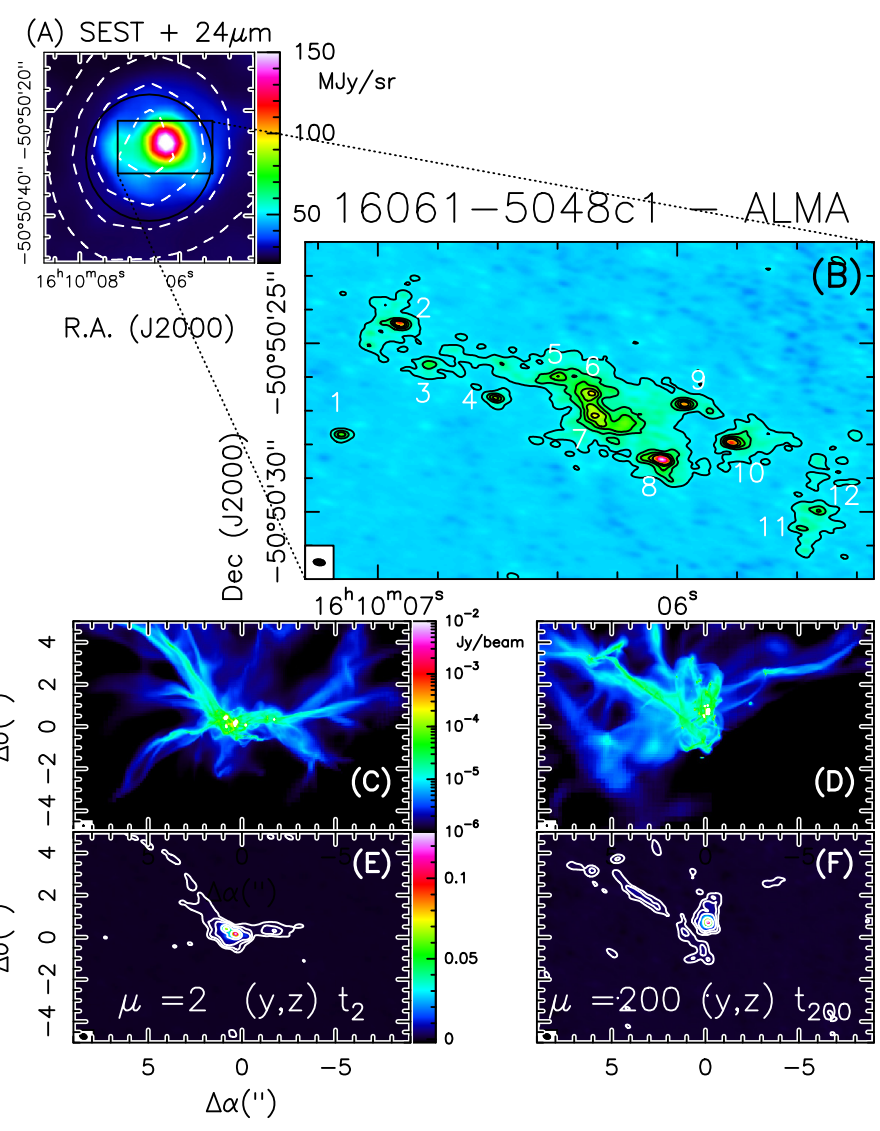

Fig. 1. Panel A): dust continuum emission map (dashed contours) obtained with the SEST telescope with an angular resolution of $24^{\prime \prime}$ at $1.2 \mathrm{~mm}$ towards I16061c1 (Beltrán et al. 2006). The map is superimposed on the Spitzer-MIPS image at $24 \mu \mathrm{m}$ (in units of MJy/sr). The circle indicates the ALMA primary beam at $278 \mathrm{GHz}\left(\sim 24^{\prime \prime}\right)$. Panel B): enlargement of the rectangular region indicated in panel A), showing the contour map of the thermal dust continuum emission at frequency $278 \mathrm{GHz}$ detected with ALMA, in flux density units. The first contour level and the spacing between two adjacent contours both correspond to the $3 \sigma \mathrm{rms}$ of the image $(0.54 \mathrm{mJy} / \mathrm{beam})$. The cross marks the phase centre. The ellipse in the bottom left corner shows the synthesized beam, and corresponds to $0.36^{\prime \prime} \times 0.18^{\prime \prime}\left(\right.$ Position Angle $\left.=86^{\circ}\right)$. The numbers indicate the twelve identified fragments (see Sect. 3). Panel C): simulations of the thermal dust emission at $278 \mathrm{GHz}$ predicted by the models of Commerçon et al. (2011), which reproduce the gravitational collapse of a $300 M_{\odot}$ clump in case of strong magnetic support $(\mu=2)$, obtained at time $t_{2}$ (see text), projected on a plane perpendicular to the direction of the magnetic field. Panel D): same as panel C) for the case $\mu=200$ (weak magnetic support). Panel E): synthetic ALMA images of the models presented in panel $\mathbf{C}$ ). The contours correspond to 0.54 , $1.2,2,5,10,30$, and $50 \mathrm{mJy} /$ beam. Panel F) same as panel E) for the case $\mu=200$ at time $t_{200}$ (weak magnetic support).

precipitable water vapour during observations was $\sim 1.8 \mathrm{~mm}$. Bandpass and phases were calibrated by observing J1427-4206 and J1617-5848, respectively. The absolute flux scale was set through observations of Titan and Ceres. From Beltrán et al. (2006), we know that the total flux measured with the single-dish in an area corresponding to the ALMA primary beam at the observing frequency $\left(24^{\prime \prime}\right)$ is $\sim 2.3 \mathrm{Jy}$, while the total flux measured with ALMA in the same area is $0.63 \mathrm{Jy}$. This means that we recover $\sim 30 \%$ of the total flux. The remaining $\sim 70 \%$ is likely contained in an extended envelope that is resolved out. Continuum was extracted by averaging in frequency the line-free channels. The total bandwidth used is $\sim 1703 \mathrm{MHz}$. 
Calibration and imaging was performed with the CASA ${ }^{1}$ software (McMullin et al. 2007), and the final images were analysed following standard procedures with the software MAPPING of the GILDAS ${ }^{2}$ package. The angular resolution of the final image is $\sim 0.25^{\prime \prime}$ (i.e. $\sim 900 \mathrm{AU}$ at the source distance). We were sensitive to point-like fragments of $0.06 M_{\odot}$. Together with the continuum, we detected several lines, among which $\mathrm{N}_{2} \mathrm{H}^{+}(3-2)$. These data will be presented and discussed in a forthcoming paper. In this letter, we only use the $\mathrm{N}_{2} \mathrm{H}^{+}(3-2)$ line to derive the virial masses, as we show in Sect. 3 .

\section{Results}

The ALMA map of the dust thermal continuum emission is shown in Fig. 1B: we have detected several dense condensations distributed in a filamentary-like structure extended eastwest, surrounded by fainter extended emission. This structure has been decomposed into twelve fragments (Fig. 1B). The fragments were identified following these criteria: (1) peak intensity greater than five times the noise level; (2) two partially overlapping fragments are considered separately if they are separate at their half-peak intensity level. The minimum threshold of five times the noise was adopted because some peaks at the edge of the primary beam are comparable to about four to five times the noise level. We decided to use these criteria and decompose the map into cores by eye instead of using decomposition algorithms (such as Clumpfind) because small changes in their input parameters could lead to large changes in the number of identified clumps (Pineda et al. 2009). The main physical properties of the fragments derived from the continuum map, that is, integrated and peak flux, size, and gas mass, and the methods used to derive them, are described in Appendix A. The derived parameters are listed in Table A.1. The mean mass of the fragments is 4.4 $M_{\odot}$, with a lowest value of $0.7 M_{\odot}$ and a highest value of $\sim 9 M_{\odot}$. The diameters (undeconvolved for the beam) range from 0.011 to $0.032 \mathrm{pc}$, with a mean value of $0.025 \mathrm{pc}$.

To investigate the stability of the fragments, we calculated the virial masses $M_{\mathrm{vir}}$, that is, the masses required for the cores to be in virial equilibrium, from the line widths observed in $\mathrm{N}_{2} \mathrm{H}^{+}(3-2)$. As stated in Sect. 2, we here used this molecular transition only for the purpose to derive the level of turbulence (the key ingredient of the models, together with the magnetic support) of the dense gas out of which the fragments are formed. The approach used to derive $M_{\text {vir }}$ is described in Appendix A and the values obtained are reported in Table A.1. The average ratio between $M_{\text {vir }}$ and $M$ computed from the continuum emission is about 0.4 , indicating that the gravity dominates, according to other ALMA studies of fragmentation (Zhang et al. 2015). However, the uncertainties due to the dust mass opacity coefficient (see Eq. (A.1)) can be of a factor 2-3, hence it is difficult to conclude that the fragments are unstable. Moreover, the formula of the virial mass we used does not consider the magnetic support. Because this latter is expected to be relevant, it is likely that the fragments are closer to virial equilibrium and would not tend to fragment further. If one assumes, for instance, the value of $0.27 \mathrm{mG}$ measured by Pillai et al. (2015) in another infrared-dark cloud, the ratio between virial mass and gas mass would become about 1 , and the fragments would be marginally stable. A similar conclusion is given in Tan et al. (2013), where the dynamics of four infrared-dark clouds similar to I16061c1 is studied.

\footnotetext{
1 The Common Astronomy Software Applications (CASA) software can be downloaded at http://casa.nrao.edu

2 http://www.iram. fr/IRAMFR/GILDAS
}

\section{Discussion and conclusion}

We have simulated the gravitational collapse of I16061c1 through 3D numerical simulations following Commerçon et al. (2011), adopting a mass, temperature, average density, and level of turbulence of the parent clump very similar to those measured (Beltrán et al. 2006; Giannetti et al. 2013). In particular, the Mach number setting the initial turbulence was derived from the line width of $\mathrm{C}^{18} \mathrm{O}$ (3-2) by Fontani et al. (2012). Because these observations were obtained with angular resolution of $\sim 24^{\prime \prime}$ and the critical density of the line $\left(\sim 5 \times 10^{4} \mathrm{~cm}^{-3}\right.$, Fontani et al. 2005) is comparable to the average density of the clump as a whole (Beltrán et al. 2006), the $\mathrm{C}^{18} \mathrm{O}$ line width represents a reasonable estimate of the intrinsic turbulence of the parent clump. The models were run for $\mu=2$ (strongly magnetised case) and $\mu=200$ (weakly magnetised case). Then, we post-processed the simulations data and computed the dust emission maps at $278 \mathrm{GHz}$ : the final maps obtained in flux density units at the distance of the source were imaged with the CASA software to reproduce synthetic images with the same observational parameters as those of the observations. A detailed description of the parameters used for the numerical simulations, of the resulting maps, and how they were post-processed is given in Appendix B. Further descriptions of the models can also be found in Commerçon et al. (2011). To investigate possible effects of geometry, we imaged the outcome of the simulations projected on three planes: $(x, y),(x, z)$, and $(y, z)$, where $x$ is the direction of the initial magnetic field. As an example, in Figs. 1C and $\mathrm{D}$ we show the results for the cases of strong and weak magnetic support, respectively, projected on the $(y, z)$ plane, that is, on a direction perpendicular to the magnetic field. The synthetic images obtained with CASA are shown in panels E and F. All the planes for $\mu=2$ and $\mu=200$ are shown in Appendix $B$, in Figs. B.1 and B.2, respectively. An important result of the simulations (see Fig. B.3 in Appendix B) is that the total flux seen by the interferometer in the $\mu=2$ case decreases until about $35 \times 10^{3} \mathrm{yr}$ and then goes through a minimum and gradually starts to increase. In the $\mu=200$ case, by contrast, the decrease is not reversed. We conclude from this that in the $\mu=2$ case, the fragments continue to accrete material and eventually will reach the total flux observed in the ALMA image. Thus, in the $\mu=2$ case we analysed the synthetic images produced at the time at which the total flux in the fragments matches the observed value within an uncertainty of about $10 \%$ (the calibration uncertainty on the flux density, see the ALMA Technical Handbook $^{3}$ ), while for the $\mu=200$ case we analysed the synthetic images obtained at the end of the simulations. This corresponds to two different times: $t_{2}=48500 \mathrm{yr}$ after the birth of the first protostar for $\mu=2 ; t_{200}=59500 \mathrm{yr}$ after the birth of the first protostar for $\mu=200$.

The synthetic maps with $\mu=200$ show more fragments with lower peak fluxes, and the overall structure is more chaotic and never filamentary, independently of the projection plane. The fragments were identified and their properties in the synthetic images derived following the same criteria and procedures as those used for the ALMA data. Therefore, any systematic error introduced by the assumptions made (e.g. the assumed dust temperature, gas-to-dust ratio, dust grain emissivity) are the same in the real and synthetic images and consequently do not affect the comparison. The statistical properties of the synthetic core populations are summarised in Table B.1 of Appendix B. We also compared the cumulative distribution of the peak fluxes of the

\footnotetext{
https://almascience.eso.org/proposing/ technical-handbook
} 

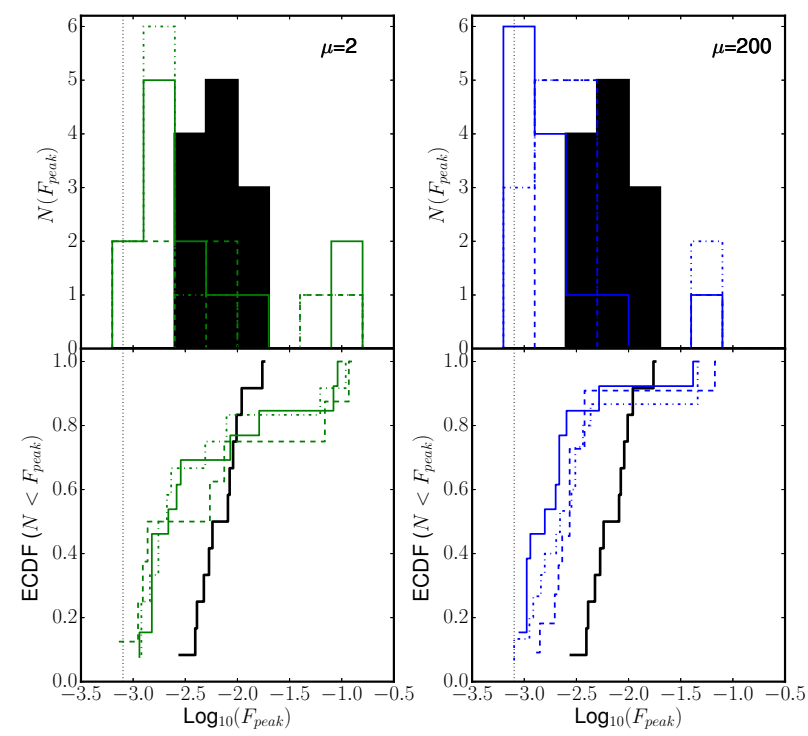

Fig. 2. Top panels: histograms showing the distribution of the peak fluxes $\left(F_{\text {peak }}\right)$ of the fragments identified in the ALMA image of I16061 1 (black), and in the synthetic images for $\mu=2$ (green, left) and $\mu=200$ (blue, right). Bottom panels: empirical cumulative distribution function (ECDF) of the quantities plotted in the top panels. The black line corresponds to the ALMA data; the green and blue lines indicate the strong and weak field cases, respectively, projected on the three planes. In all panels, the different line style indicates the projection plane: solid $=(x, y)$; dot-dashed $=(x, z)$; dashed $=(y, z)$. The vertical dotted line corresponds to $0.8 \mathrm{mJy}$, which is approximately five times the rms noise level in both the real and synthetic maps. Note that the $\mu=2$ model spans the observations, while the $\mu=200$ model is strongly biased towards fragments with masses lower than those observed.

fragments in the observed and synthetic images. The results are shown in Fig. 2. The case with $\mu=200$ has lower peak values for the whole populations, while one or two fragments have a higher peak value than the maximum observed. The $\mu=2$ case has a broader distribution of values. Overall, the ALMA map shows a narrower distribution of peak fluxes, with a deficit of very weak and very strong peaks, which in turn are present in the two synthetic images. However, the $\mu=2$ model roughly spans the observations, while the $\mu=200$ model is heavily biased below the data. In addition, a non-parametric statistical test (Anderson-Darling test) implies that all the $\mu=200$ cases can be excluded as being drawn from the same parent distribution as the observed values with a confidence level exceeding $99.8 \%$. The $\mu=2$ case is less obvious because two projections could be excluded at a 98-99\% confidence level, while the third projection, $(y, z)$, with a null hypothesis probability of $\sim 90 \%$ cannot be excluded at the $2 \sigma$ level. The deficit of very strong and very weak peaks in the real image may be due to a difference between the $\mu$ values assumed in the simulations and the real one, or to some other unknown (or doubtful) initial assumption such as the density profile or the homogeneous temperature of the collapsing clump.

Based on the overall morphologies shown in Fig. 1 (and in Figs. B.1 and B.2) and on the statistical properties of the fragments reported in Fig. 2, the model that better reproduces the data clearly is the one with $\mu=2$. Hence, with these new ALMA observations, compared with realistic $3 \mathrm{D}$ simulations that assume as initial conditions the properties of the parent clump, we demonstrate that the fragmentation due to self-gravity is dominated by the magnetic support, based on the evidence that (1) the overall morphology of the fragmenting region is filamentary, and this is predicted only in case of a dominant magnetic support; (2) the observed fragment mass distribution is most easily understood in simulations assuming substantial magnetic support.

Acknowledgements. This paper makes use of the following ALMA data: ADS/JAO.ALMA.2012.1.00366.S. ALMA is a partnership of ESO (representing its member states), NSF (USA) and NINS (Japan), together with NRC (Canada), NSC and ASIAA (Taiwan), and KASI (Republic of Korea), in cooperation with the Republic of Chile. The Joint ALMA Observatory is operated by ESO, AUI/NRAO and NAOJ We acknowledge the Italian-ARC node for their help in the reduction of the data. We acknowledge partial support from Italian Ministero dell'Istruzione, Università e Ricerca through the grant Progetti Premiali 2012 - iALMA (CUP C52I13000140001) and from Gothenburg Centre of Advanced Studies in Science and Technology through the program Origins of habitable planets.

\section{References}

Bate, M. R. 2009, MNRAS, 392, 1363

Beltrán, M. T., Brand, J., Cesaroni, R., et al. 2006, A\&A, 447, 221

Beuther, H., Schilke, P., Sridharan, T. K., et al. 2002, A\&A, 566, 945

Bleuler, A., \& Teyssier, R. 2014, MNRAS, 445, 4015

Bonnell, I. A., Vine, S. G., \& Bate M. R. 2004, MNRAS, 349, 735

Bontemps, S., Motte, F., Csengeri, T., \& Schneider, N. 2010, A\&A, 524, A18

Caselli, P., Walmsley, C. M., Tafalla, M., Dore, L., \& Myers, P. 1999, ApJ, 523, L165

Commerçon, B., Hennebelle, P., \& Henning, T. 2011, ApJ, 742, L9

Commerçon, B., Launhardt, R., Dullemond, C., \& Henning, Th. 2012, A\&A, 545, A98

Commerçon, B., Debout, V., \& Teyssier, R. 2014, A\&A, 563, A11

Crutcher, R. M. 2013, ARA\&A, 50, 29

Dobbs, C. L., Bonnell, I. A., \& Clark, P. C. 2005, MNRAS, 360, 2

Emprechtinger, M., Caselli, P., Volgenau, N. H., Stutzki, J., \& Wiedner, M.C. 2009, A\&A, 493, 89

Fontani, F., Beltrán, M. T., Brand, J., et al. 2005, A\&A, 432, 921

Fontani, F., Giannetti, A., Beltrán, M. T., et al. 2012, MNRAS, 423, 2342

Fromang, S., Hennebelle, P., \& Teyssier, R. 2006, A\&A, 457, 371

Giannetti, A., Brand, J., Sanchez-Monge, Á., et al. 2013, A\&A, 556, A16

Hennebelle, P., Commerçon, B., Joos, M., et al. 2011, A\&A, 528, A72

Kauffmann, J., \& Pillai, T. 2010, ApJ, 723, L7

Krumholz, M. R. 2006, ApJ, 641, L45

Krumholz, M. R., Klein, R., McKee, C. F., Offner, S. S. R., \& Cunningham, A. J. A. 2009, Science, 323, 754

Longmore, S. N., Pillai, T., Keto, E., Zhang, Q., \& Qiu, K. 2011, ApJ, 726, L97

Lopez-Sepulcre, A., Cesaroni, R., \& Walmsley, C. M. 2010, A\&A, 517, A66

Maret, S., Hily-Blant, P., Pety, J., Bardeau, S., \& Reynier, E. 2011, A\&A, 526, A47

McKee, C., \& Tan, J. C. 2003, ApJ, 585, 850

McMullin, J. P., Waters, B., Schiebel, D., Young, W., \& Golap, K. 2007, in

Astronomical Data Analysis Software and Systems XVI, eds. R. A. Shaw,

F. Hill, \& D. J. Bell, ASP Conf. Ser., 376, 127

Mouschovias, T. C., \& Spitzer, L. J. 1976, ApJ, 210, 326

Ossenkopf, V., \& Henning, T. 1994, A\&A, 291, 943

Palau, A., Fuente, A., Girart, J. M., et al. 2013, ApJ, 762, 120

Pillai, T., Kauffmann, J., Tan, J. C., et al. 2015, ApJ, 799, 74

Pineda, J. E., Rosolowsky, E. W., \& Goodman, A. A. 2009, ApJ, 699, L134

Sanchez-Monge, Á, Beltrán, M. T., Cesaroni, et al. 2013, A\&A, 550, A21

Tan, J. C., Kong, S., Butler, M. J., Caselli, P., \& Fontani, F. 2013, ApJ, 779, 96

Teyssier, R. 2002, A\&A, 385, 337

Weiss, A., Hillebrandt, W., Thomas, H.-C., \& Ritter, H. 2005, Book review:

Cox and Giuli's Principles of Stellar Structure (Cambridge, UK: Princeton Publishing Associates Ltd)

Zhang, Q., Wang, K., Xing, L., \& Jiménez-Serra, I. 2015, ApJ, 804, 141 
Table A.1. Peak position (in RA and Dec J2000), integrated flux (inside the $3 \sigma \mathrm{rms}$ contour level), peak flux, diameter, mass, line width at half maximum, and virial mass of the 12 fragments identified in Fig. 1B.

\begin{tabular}{lcccccccc}
\hline \hline Fragment & $\begin{array}{c}\text { RA (J2000) } \\
\text { h:m:s }\end{array}$ & $\begin{array}{c}\text { Dec (J2000) } \\
\text { deg: : }:^{\prime \prime}\end{array}$ & $\begin{array}{c}S_{v} \\
\mathrm{Jy}^{2}\end{array}$ & $\begin{array}{c}S_{v}^{\text {peak }} \\
\mathrm{Jy} \mathrm{beam}^{-1}\end{array}$ & $\begin{array}{c}D \\
\mathrm{pc}\end{array}$ & $\begin{array}{c}M \\
M_{\odot}\end{array}$ & $\begin{array}{c}\Delta v \\
\mathrm{~km} \mathrm{~s}^{-1}\end{array}$ & $\begin{array}{c}M_{\text {vir }} \\
M_{\odot}\end{array}$ \\
\hline 1 & $16: 10: 07.12$ & $-50: 50: 27.7$ & 0.007 & 0.0054 & 0.011 & 0.72 & 0.51 & 0.28 \\
2 & $16: 10: 06.93$ & $-50: 50: 24.4$ & 0.045 & 0.0092 & 0.031 & 4.70 & 0.90 & 1.86 \\
3 & $16: 10: 06.83$ & $-50: 50: 25.6$ & 0.012 & 0.0041 & 0.018 & 1.25 & 0.48 & 0.47 \\
4 & $16: 10: 06.60$ & $-50: 50: 26.6$ & 0.012 & 0.0058 & 0.016 & 1.25 & 0.82 & 1.26 \\
5 & $16: 10: 06.40$ & $-50: 50: 26.0$ & 0.044 & 0.0048 & 0.029 & 4.59 & 0.49 & 0.77 \\
6 & $16: 10: 06.29$ & $-50: 50: 26.5$ & 0.073 & 0.0086 & 0.028 & 7.62 & 0.36 & 0.38 \\
7 & $16: 10: 06.27$ & $-50: 50: 27.1$ & 0.050 & 0.0083 & 0.023 & 5.22 & 0.33 & 0.26 \\
8 & $16: 10: 06.16$ & $-50: 50: 27.3$ & 0.052 & 0.0180 & 0.024 & 5.42 & 0.84 & 2.01 \\
9 & $16: 10: 05.98$ & $-50: 50: 26.8$ & 0.045 & 0.010 & 0.029 & 4.70 & 0.33 & 0.33 \\
10 & $16: 10: 06.05$ & $-50: 50: 28.4$ & 0.084 & 0.011 & 0.031 & 8.76 & 1.04 & 3.47 \\
11 & $16: 10: 05.82$ & $-50: 50: 27.9$ & 0.052 & 0.0028 & 0.032 & 5.43 & 1.00 & 3.29 \\
12 & $16: 10: 05.53$ & $-50: 50: 30.0$ & 0.032 & 0.0041 & 0.030 & 3.34 & 0.76 & 1.82 \\
\hline
\end{tabular}

Notes. The line widths are computed from the $\mathrm{N}_{2} \mathrm{H}^{+}(3-2)$ spectra extracted from the polygons defining the external profile of each fragment, as explained in Appendix A.

\section{Appendix A: Physical properties of the fragments}

\section{A.1. Derivation of the parameters}

- Integrated flux densities: the integrated flux densities of the fragments, $S_{v}$, were obtained from the $3 \sigma$ rms contour in the continuum image. In the few cases for which the $3 \sigma$ rms contours of two adjacent fragments are partly overlapping (e.g. fragments 5, 6, and 7 in Fig. 1), the edges between the two have been defined by eye at approximately half of the separation between the peaks. The results are shown in Table A.1.

- Gas masses: the gas mass of each fragment was calculated from the equation

$M=\frac{g S_{v} d^{2}}{\kappa_{v} B_{v}\left(T_{\mathrm{d}}\right)}$,

where $S_{v}$ is the integrated flux density, $d$ is the distance to the source, $\kappa_{v}$ is the dust mass opacity coefficient, $g$ is the gasto-dust ratio (assumed to be 100), and $B_{v}\left(T_{\mathrm{d}}\right)$ is the Planck function for a black body of temperature $T_{\mathrm{d}}$. We adopted $T_{\mathrm{d}}=25 \mathrm{~K}$, corresponding to the gas temperature derived by Giannetti et al. (2013), assuming coupling between gas and dust (reasonable assumption at the high average density of the clump). The dust mass opacity coefficient was derived from the equation $\kappa_{v}=\kappa_{v_{0}}\left(v / v_{0}\right)^{\beta}$. We assumed $\beta=2$ and $\kappa_{v_{0}}=0.899 \mathrm{~cm}^{-2} \mathrm{gr}^{-1}$ at $\nu_{0}=230 \mathrm{GHz}$, according to Ossenkopf \& Henning (1994). The highest mass derived is $\sim 9 M_{\odot}$, the lowest is $\sim 0.7 M_{\odot}$ (see Table A.1).

- Size: the size of each fragment was estimated as the diameter of the circle with an area equivalent to that encompassed by the $3 \sigma$ rms contour level. The results are shown in Table A.1. The ALMA beam size is much smaller than the size of the fragments, so that deconvolution for the beam is irrelevant to derive the source size.

- Virial masses: the virial masses were derived in this way: first, we extracted the $\mathrm{N}_{2} \mathrm{H}^{+}$(3-2) spectra from the $3 \sigma \mathrm{rms}$ level of the 12 continuum cores. All the spectra were fitted in an automatic way using a procedure based on the integration of the python module PyMC and the CLASS extension WEEDS (Maret et al. 2011). Then, the virial masses, $M_{\text {vir }}$, were computed from the formula

$M_{\mathrm{vir}}=210 r \Delta v^{2} M_{\odot}$, where $r$ is the size of the fragment, and $\Delta v$ is the line width at half maximum of the average $\mathrm{N}_{2} \mathrm{H}^{+}(3-2)$ spectrum obtained from the fitting procedure described above. The results are shown in Table A.1.

\section{Appendix B: Simulations and synthetic images}

\section{B.1. Methods and initial conditions for the numerical calculations}

We performed a set of two radiation-magneto-hydrodynamics calculations that includes the radiative feedback from the accreting protostars. We used the RAMSES code with the grey flux-limited-diffusion approximation for radiative transfer and the ideal magnetohydrodynamics (MHD) for magnetic fields (Commerçon et al. 2012, 2014; Teyssier 2002; Fromang et al. 2006). The initial conditions are similar to those used in Hennebelle et al. (2011) and Commerçon et al. (2011), with slight modifications to match roughly the observed properties of $116061 \mathrm{c} 1$. We note that the models presented in this Letter were made with initial conditions very similar to those measured from previous observations in I16061c1to perform an appropriate comparison with observations for this specific source. Our aim was not to fine-tune the initial conditions such that the models best reproduce the observations. We considered an isolated spherical core of mass $300 M_{\odot}$, radius $0.25 \mathrm{pc}$, and temperature $20 \mathrm{~K}$. We assumed a Plummer-like initial density profile $\rho(r)=\rho_{c} /\left(1+\left(r / r_{0}\right)^{2}\right)$, with $\rho_{c}=3.96 \times 10^{5} \mathrm{~cm}^{-3}$ and $r_{0}=0.085 \mathrm{pc}$, and a factor 10 for the density contrast between the centre and the border of the core. This density profile is suggested by observational findings (Beuther et al. 2002). The initial magnetic field was aligned with the $x$-axis and its intensity was proportional to the column density through the cloud (Hennebelle et al. 2011). We here investigated two degrees of magnetization, $\mu=2$, which is close to the values $2-3$ that are observationally inferred (e.g. Crutcher 2012), and $\mu=200$, which corresponds to a quasi-hydrodynamical case. Last, we applied an initial internal turbulent velocity field to the cores. The velocity field was obtained by imposing a Kolmogorov power spectrum with randomly determined phases (i.e. a ratio $2: 1$ between the solenoidal and the compressive modes). There is no 
global rotation of the cloud, meaning that the angular momentum is contained within the initial turbulent motions, which are then amplified by the gravitational collapse. The amplitude of the velocity dispersion was scaled to match a turbulent Mach number of 6.44, in agreement with $\mathrm{C}^{18} \mathrm{O}$ observations (Fontani et al. 2012). Following Hennebelle et al. (2011, Eq. (2) therein), the virial parameter was $\alpha_{\text {vir }} \sim 0.72$ for $\mu=2$, and $\alpha_{\text {vir }} \sim 0.54$ for $\mu=200$ (close to virial equilibrium in both cases). The two calculations, $\mu=2$ and $\mu=200$, started with the same initial turbulent velocity field (only one realisation was explored) and turbulence was not maintained during the collapse. Investigation of the effect of different initial turbulent seeds is beyond the scope of this Letter. The computational box had a 2563 resolution, and the grid was refined according to the local Jeans length (at least 8 cells/Jeans length) down to seven levels of refinement (minimum cell size of $13 \mathrm{AU}$ ). Below $13 \mathrm{AU}$, the collapsing gas was described using sub-grid models attached to sink particles, similar to what was done in other studies (Krumholz et al. 2009). We used the sink particle method presented in Bleuler et al. (2014), although with slight modifications on the checks performed for the sink creation. The sink particles accrete the gas that is located in their accretion volumes (sphere of radius $\sim 52 \mathrm{AU}, 4$ cells) and that is Jeans unstable. We considered that half of the mass accreted onto the sink particles becomes stellar material. The luminosity of the protostars was then computed using mass-radius and mass-luminosity empirical relations of main-sequence stars (e.g. Weiss et al. 2005) and was injected within the accretion volume in the computational domain as a source term (e.g. Krumholz et al. 2009). We did not account for accretion luminosity.

\section{B.2. Outcomes of the numerical calculations}

We ran the calculations until they reached a star formation efficiency $(S F E)>20 \%$ (where the star formation efficiency corresponds to the ratio between the mass of the gas accreted onto the sink particles and the total mass of the cloud). Again, the choice of the times at which we stopped the calculations was not aimed at best reproducing the observed values. Model $\mu=2$ is post-processed at time $t_{2}=48500 \mathrm{yr}$ after the birth of the first protostar, which is the time at which the total flux in fragments is equal to the observed value (within the uncertainty), and $\mu=200$ at time $t_{200}=59500 \mathrm{yr}$. At these times, model $\mu=2$ has formed 38 sink particles (for a total mass of $60 M_{\odot}$ ), while model $\mu=200$ has formed 119 sink particles (for a total mass of $85 M_{\odot}$ ). Figure B.3 shows the time evolution after the first sink creation of the SFE, the number of sinks, and the total flux at $278 \mathrm{GHz}$ (within a total area of $80000 \mathrm{AU} \times 80000 \mathrm{AU}$ ) for the two models. The circles indicate the time at which the simulations are post-processed. We note the increase in the $278 \mathrm{GHz}$ flux with time for $\mu=2$, which probably reflects the temperature increase caused by the radiation of the sink particles.

\section{B.3. Production of the synthetic images}

We first post-processed the RAMSES calculation results using the radiative transfer code $\mathrm{RADMC}-3 \mathrm{D}^{4}$ and the interface presented in Commerçon et al. (2012). We produced dust emission maps at $278 \mathrm{GHz}$ (see Figs. B.1 and B.2). We did not account for the stellar luminosities in the synthetic images since the

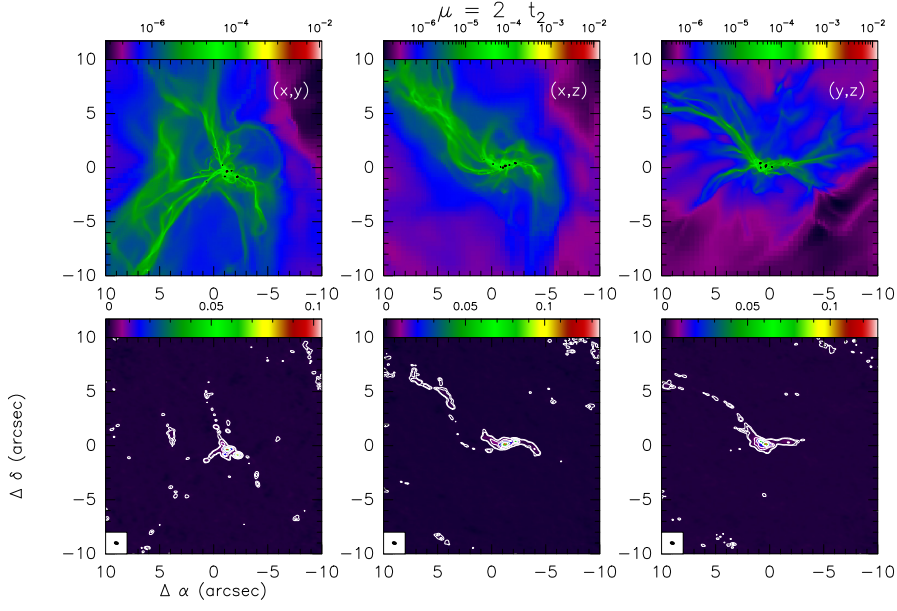

Fig. B.1. Top panels: show the thermal dust continuum emission map at frequency $278 \mathrm{GHz}$ predicted by the models of Commerçon et al. (2011), which reproduce the gravitational collapse of a $300 M_{\odot}$ clump in case of strong magnetic support $(\mu=2)$ at time $t_{2}=48500 \mathrm{yr}$ after the birth of the first protostar (see main text for details). In the bottom panels, we show the models after processing in the CASA simulator, adopting the same observational conditions as for the real observations. Units of the colour-scale are Jansky/beam. Contour levels are $0.6,1,5,10,30$, and $50 \mathrm{mJy}^{-1}$ beam $^{-1}$ in all bottom panels.

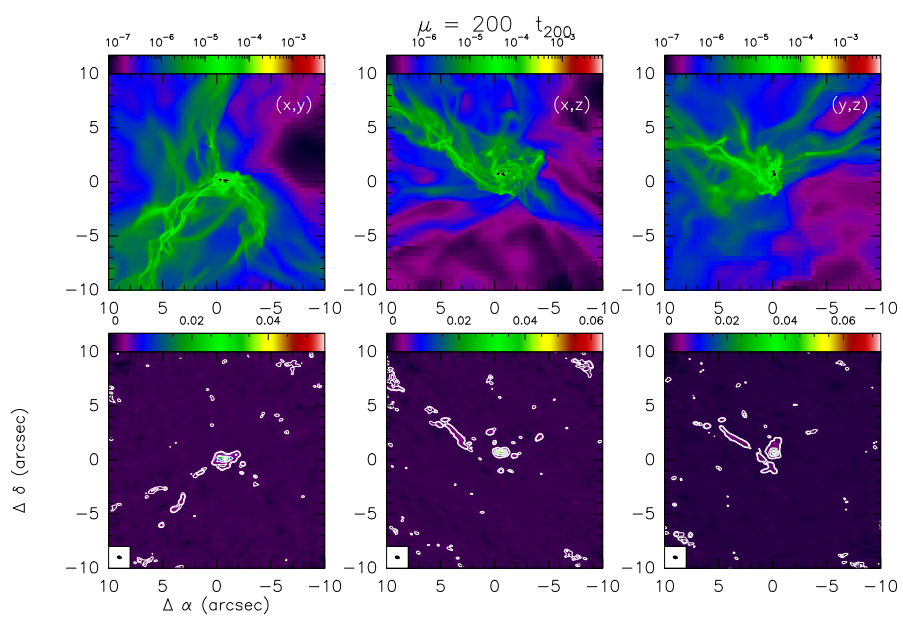

Fig. B.2. Same as Fig. B. 1 for the case $\mu=200$ at time $t_{200}=59500 \mathrm{yr}$ after the birth of the first protostar (see main text for details).

stellar irradiation is reprocessed in the envelope at millimeter wavelengths. However, we attempted to create models accounting for protostellar luminosities, and found results that do not change significantly at the wavelength considered. The ALMA synthetic images of the numerical simulations were then produced through the CASA software: first, synthetic visibilities were created with the task simobserve, which were then imaged with the task simanalyze. To precisely reproduce the observations, we used the same parameters in the tasks as in the observations: integration time on source of $18 \mathrm{~min}$, precipitable water vapour of about $1.8 \mathrm{~mm}$, array configuration C36-6, start hour angle of $2.4 \mathrm{~h}$ (see Sect. 2). The population of fragments in the final synthetic images were derived following the same procedure as described in Sect. 4.

\footnotetext{
4 http://www.ita.uni-heidelberg.de/dullemond/ software/radmc-3d/
} 
F. Fontani et al.: Magnetically regulated fragmentation
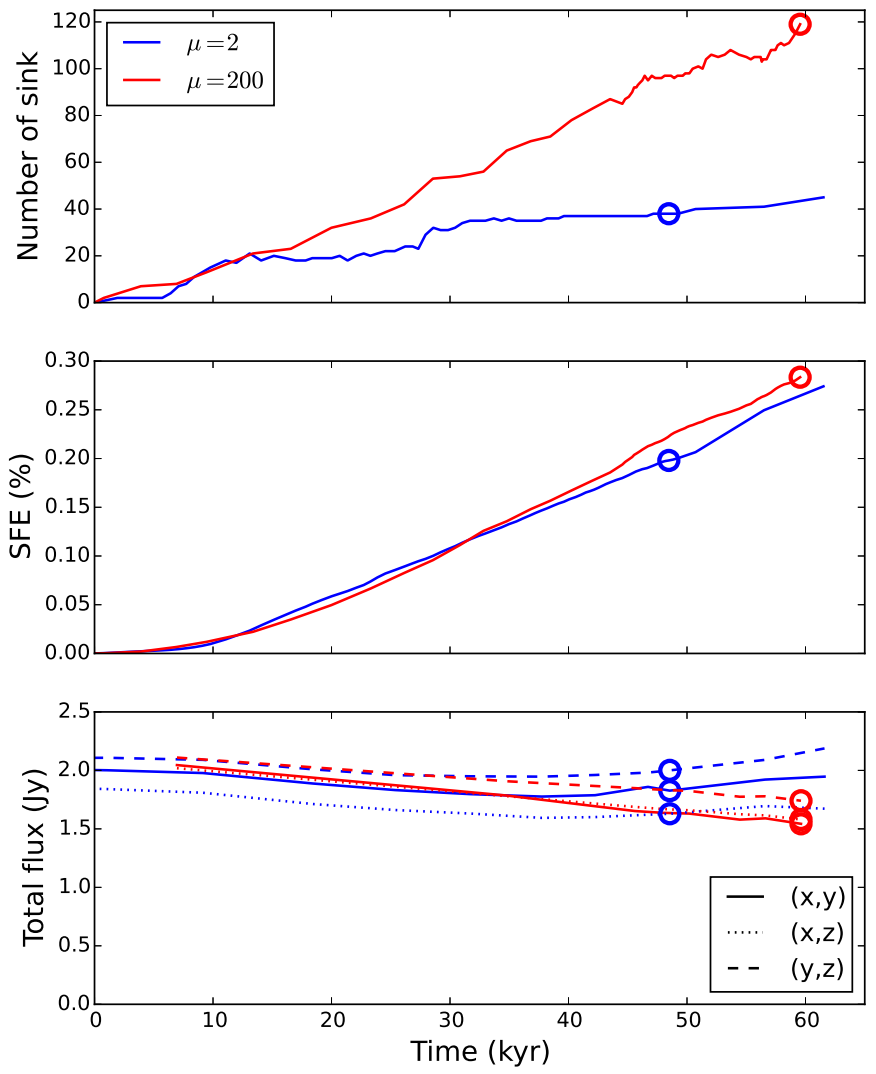

Fig. B.3. From top to bottom: evolution with time of the number of sink particles, of the SFE, and of the total flux emission at $278 \mathrm{GHz}$ (within a total area of $80000 \mathrm{AU} \times 80000 \mathrm{AU}$ ) for the two models after the creation of the first sink. The circles indicate the time at which the simulations are post-processed. In the bottom panel, the different lines correspond to the different projection planes as illustrated in the bottom right corner.
Table B.1. Statistical comparison between the fragment population derived from the ALMA image of I16061c1 shown in Fig. 1 and the simulations presented in Figs. B1 and B2 of the Appendix.

\begin{tabular}{lcccccc}
\hline \hline & $\begin{array}{c}S_{v}^{\text {tot }} \\
\text { Jy }\end{array}$ & $\begin{array}{c}M^{\text {tot }} \\
M_{\odot}\end{array}$ & $N$ & $\begin{array}{c}D_{\text {mean }} \\
\mathrm{pc}\end{array}$ & $\begin{array}{c}S_{v}^{\text {mean }} \\
\text { Jy }\end{array}$ & $\begin{array}{c}M^{\text {mean }} \\
M_{\odot}\end{array}$ \\
\hline ALMA & 0.52 & 53 & 12 & 0.025 & 0.042 & 4.42 \\
$\mu=2(x, y)$ & 0.36 & 36 & 12 & 0.013 & 0.026 & 2.76 \\
$\mu=2(x, z)$ & 0.47 & 49 & 12 & 0.017 & 0.039 & 4.1 \\
$\mu=2(y, z)$ & 0.46 & 42 & 8 & 0.018 & 0.050 & 5.2 \\
$\mu=200(x, y)$ & 0.22 & 23 & 13 & 0.015 & 0.017 & 1.74 \\
$\mu=200(x, z)$ & 0.24 & 25 & 15 & 0.014 & 0.016 & 1.67 \\
$\mu=200(y, z)$ & 0.28 & 24 & 16 & 0.016 & 0.021 & 2.19 \\
\hline
\end{tabular}

Notes. The derivation of the parameters obtained for the observed and synthetic images is described in Sect. 3 and in Appendix A, respectively. 